\title{
Disease insights from stem cells
}

Confronted by religious and political opposition, proponents of embryonic-stem-cell research need to draw attention not only to new therapies but also to the understanding of diseases that they could soon yield.

A t a symposium on stem cells, regenerative medicine and cancer held last week at Stanford University in California, leading stem-cell researchers argued that the main effect of the technology on biomedical research will be felt not in cell-replacement therapies, but in applications that have until now received little attention: the use of embryonic stem (ES) cells in uncovering the mechanisms of genetic diseases, and in generating sources of normal and impaired tissues for use in drug discovery.

Human ES cells are derived from five-day-old human embryos, and their ability to grow and expand in an unlimited manner, and to form an array of specialized mature cell types upon command, has made them a valuable, albeit controversial, tool for biomedical research. A beautiful example of the power and limitations of ES cells was presented at the meeting by Hynek Wichterle, who works with Thomas Jessell at Columbia University in New York. He showed that mouse ES cells can be induced systematically to form motor neurons, the cell type that withers and dies in patients suffering from the fatal and incurable disease amyotrophic lateral sclerosis (ALS).

When he transplanted the motor neurons into mice, the cells survived and grew but failed to establish the appropriate connections within the mouse's nervous system. Nonetheless, the plates loaded with this precious cell type provide, for the first time, a plentiful and renewable source of motor neurons for identifying therapeutic molecules that promote motor-neuron growth, and prevent their death. Other scientists have developed protocols for inducing mouse and human ES cells to form a variety of tissues.

But even more powerful than generating healthy tissues from the existing human ES-cell lines would be the ability to generate new cell lines that could produce unlimited quantities of diseased tissue where the disease has a genetic basis, such as motor neurons from ALS patients, or cardiac muscle cells from heart patients. Scientists could then use the cell lines to study the cellular processes that go awry in the diseased cells, and design high-throughput screens to identify molecules that halt disease progression. It is feasible to generate ES-cell lines from mouse models of human disease, but often the underlying causes may be different from those of the human diseases they are supposed to represent. And many human diseases are so complex or poorly understood that mouse models do not exist for them.

\section{Growth area}

It has not yet been possible to generate ES-cell lines from adult humans - the existing lines have all been derived from embryos left over from in vitro fertilization (IVF). But although this appears to be technically difficult, it will no doubt be worth the effort. For example, Irving Weissman, who heads the newly launched Institute for Cancer/Stem Cell Biology and Medicine at Stanford University, has made this one of the goals of the privately funded institute, and plans to generate new ES-cell lines from patients with cancer, diabetes, cardiovascular disease, autoimmune disease, allergies and neurodegenerative diseases such as Parkinson's and ALS.

Weissman argues that the existing human ES-cell lines, which are all derived from IVF embryos, do not harbour the mutations that cause these diseases and so cannot be used for studying human disease in the manner that he proposes. Moreover, the existing cell lines do not reflect the genetic diversity of the overall population, but rather that of the mainly white, infertile clientele of the clinics. These lines could not help scientists to understand, for example, why there is currently an epidemic of type II diabetes in the Native American population.

With current technologies, generating new ES-cell lines from patients would require somatic-cell nuclear transfer (SCNT). This procedure involves extracting the nucleus from a patient's mature cells ( skin cells, for example). These cells will contain virtually identical genetic information to that contained in the patient's diseased cells. The nuclei would then be transferred to an enucleated egg (oocyte), which would be stimulated to divide in a Petri dish to develop into an embryo. After about five days, the ES cells would be extracted from the blastocyst (which destroys it), cultured in vitro and induced to form the diseased tissue.

\section{Obstacles}

Although using ES-cell technology to understand the mechanisms of human disease could be spectacularly illuminating, there are practical and political hurdles in bringing this idea to fruition. The first problem is that deriving new ES cells from human embryos, even spare IVF embryos that are destined for destruction, is not allowed in the United States with public funding under current federal guidelines - although it is permitted if funds are private. It is also forbidden in several European countries.

Second, even if the technical problems of performing SCNT with human cells is overcome, there is opposition to SCNT because it involves the production of human embryos for research. Moreover, it has inappropriately been lumped into the general category of 'cloning', and so may be subject to regulation that is aimed mainly at preventing reproductive cloning. If the Brownback Bill pending in the Senate is passed, US researchers who perform this procedure could be subject to 10 years' imprisonment and a $\$ 1$-million fine. And if amendments to a safety bill last week by European parliamentarians are ratified by national governments, such procedures would be banned in Europe, along with all other techniques for producing embryos for research. In contrast, Singapore allows the use of SCNT to produce human embryos, which can then be kept for 14 days to allow ES cells to be extracted.

There are practical difficulties too. Human oocytes for SCNT are in short supply because of the invasive nature of the procedure required to harvest them, so scientists will need to find a way to collect eggs from ovaries that have been donated for research (each of which contains about 200,000 oocytes), learn to culture human oocytes from progenitor cells, or develop ways to generate human ES-cell lines without using oocytes.

Although there are numerous challenges that have yet to be overcome, a 'tipping point' will eventually be reached where technical problems will become solvable. This will result in a sea change in the field, leading to rapid progress. The implications of ES-cell technology for understanding and curing human disease are enormous. These benefits need to be fully appreciated and communicated in the midst of political and ethical debates. 